\title{
Program ACTIVE: Addressing the Need for Accessible Depression Treatment in a Rural Population with Diabetes
}

\author{
Mary de Groot
}

2009

The prevalence of type 2 diabetes (T2DM) continues to rise in epidemic proportions both in the U.S. and globally [1]. Currently within the U.S., T2DM accounts for greater than $90 \%$ of all diabetes cases and is over-represented among ethnic minority and other underserved populations, including the rural Appalachian region [2, 3]. As a region, rural Appalachian counties experience greater burdens of poverty, income inequality, and unemployment compared to nonAppalachian U.S. counties [4, 5] and exceed state and national averages.

Poverty, socioeconomic stress and consequent unhealthy lifestyles have been shown to increase the risk for the development of obesity and T2DM as well as morbidity and premature mortality of T2DM [6-10]. Recent data have demonstrated higher rates of self-reported T2DM (11.3\%) in Appalachian Ohio compared to the general population, rivaling rates found among ethnic minority communities [2]. Individuals with diabetes in this region reported a two-fold higher prevalence of heart disease and a three-fold higher prevalence of stroke compared to nondiabetes individuals [2].

This is the author's manuscript of the article published in final edited form as: 
Impact of Co-Morbid Depression

Patients with T2DM have been found to be two times more likely to experience depressive symptoms than their non-diabetes peers [11]. The aggregated lifetime prevalence of major depression has been found to range between $11.4 \%$ and $27 \%$, with greater rates found in studies using depression questionnaires [11].

Depressive symptoms have been shown to have negative consequences for individuals with diabetes. Depression has been shown to be associated with worsened blood glucose levels [12] and diabetes complications [13] such as coronary heart disease (CHD) [14]. Depression is also associated with decreased adherence to self-care regimens, increased medical costs associated with greater depression severity and greater ambulatory care use and prescription costs [15-17] in individuals with diabetes.

Despite the significant costs of co-morbid depression and diabetes, relatively few controlled depression treatment trials have been conducted. The majority of trials have demonstrated the efficacy of medication treatment [19-24]. Lustman and colleagues conducted the benchmark randomized controlled trial for cognitive behavioral therapy (CBT) in patients with co-morbid depression and diabetes [18]. In this study, 51 patients diagnosed with T2DM and major depression were randomly assigned to a 10-week individualized CBT or control condition without medication intervention. Findings showed $71 \%$ of patients receiving CBT compared to $22 \%$ of patients in the control group achieved depression remission at post-treatment assessment. At 6-month follow-up assessments, depression remitted in 67\% of patients in the CBT condition compared to $30 \%$ in the control group [18]. In addition, clinically significant reductions in 
average blood glucose (HbA1c) were found among those in the CBT condition at 6 month follow-up assessment.

No studies to date have examined the effectiveness of aerobic exercise as a treatment for major depression in patients with diabetes, although a variety of studies have demonstrated antidepressant effects of exercise in patients with clinical depression [25-28]. In randomized clinical trials, exercise was shown to be as effective as antidepressant medications in treating depression with the benefits of exercise extending beyond those observed in medication alone [25-26].

Finally, exercise interventions have been shown to improve glycemic control in patients with T2DM [29-35]. Exercise training has also been shown to result in the reduction of a variety of cardiovascular risk factors including: upper-body visceral adiposity [30], improvement in insulin sensitivity [31], increase in high density lipoprotein cholesterol [HDL-C; [32-33], reduction of triglycerides, increased LDL particle size, reduction of hypertension, and decreased total cholesterol [35-39].

Taken together, there is mounting evidence for the need to develop effective and accessible depression treatment for individuals with T2DM and depression. Program ACTIVE (Appalachians Coming Together to Increase Vital Exercise) was designed to test the effectiveness of a combination behavioral approach to the treatment of depression in adult patients with T2DM, living in the Appalachian region, on depression, glycemic and cardiovascular risk outcomes. The study received Institutional Review Board approval from Ohio University. 
The primary study aims were:

1. To assess the feasibility of recruitment, retention, and adherence of an at-risk Appalachian T2DM sample with major depression to a 12-week interdisciplinary depression treatment protocol combining CBT and community-based exercise;

2. To assess changes in depression following intervention (POST) and 3-month follow-up (3MFU) assessments compared to baseline.

3. To assess changes in glycemic control at POST and at the 3MFU assessments.

4. To assess changes in blood lipids as an index of cardiovascular risk, at POST and 3MFU. Maximal aerobic capacity (VO2 max), exercise tolerance, and resting blood pressure were evaluated at POST and 3MFU assessments.

The secondary aims of Program ACTIVE were to assess changes in quality of life and social support at POST and 3MFU compared to baseline.

\section{Study Design}

Program ACTIVE was a single-arm repeated measures intervention study conducted in two phases. In Phase I, a culturally consonant CBT manual was created. Exercise protocol materials based on the Diabetes Prevention Program [DPP; 40] were culturally-tailored for a rural Appalachian population with T2DM. All materials were evaluated for cultural salience, readability and comprehension by key informants drawn from the community and national consultants (de Groot, et al., In preparation).

In Phase II, the interdisciplinary treatment protocol was implemented. Participants were recruited from the community through direct physician referral and community advertisement. Participants 
were screened by phone for initial study inclusion before completing a baseline assessment protocol. Participants who met eligibility criteria during baseline assessment were enrolled in the intervention. Participants completed follow-up assessments immediately following the intervention (POST) and 3 months following intervention completion (3MFU).

\section{Recruitment}

Participants for Program ACTIVE were recruited from communities in southeastern Ohio and western West Virginia. In order to ensure participant safety, the following eligibility criteria were used: age 18 or older, ability to engage in walking or other physical activity, diagnosis of T2DM for one year duration or longer, and a current diagnosis of major depression with no evidence of psychotic symptoms. Individuals were excluded from participation if they were diagnosed with current or recent medical events that would preclude safe participation in moderate intensity physical activity (e.g. uncontrolled hypertension, medical instability, recent cardiac events, etc.) or met criteria for psychiatric disorders not addressed by the intervention (e.g. bipolar disorder).

A total of $\mathrm{N}=336$ individuals were screened by phone to assess medical and psychiatric eligibility. Twenty-three percent $(\mathrm{N}=65)$ of these individuals met eligibility criteria and were referred for baseline assessment. Of these, $\mathrm{N}=50$ (77\%) individuals were enrolled in the intervention.

\section{Assessment Procedure}

Psychological, behavioral and physiologic measures were administered at each of the three assessment periods: baseline, immediately following the intervention (POST) and 3-month 
follow-up assessment (3MFU). Psychological and behavioral measures included: demographic questionnaire; the Structured Clinical Interview for the DSM-IVTR [41,42] used to assess lifetime and current diagnoses of Axis I disorders; Beck Depression Inventory (BDI-II) used to assess symptoms of depression [43]; Diabetes Quality of Life Measure (DQOL) used to assess diabetes-specific quality of life [45]; SF-36 Quality of Life Measure (SF-36)used to assess general quality of life [45]; Chronic Illness Resource Survey (CIRS) used to assess social support using an ecological lens [46]; Physical Activity Diaries adapted from the DPP Lifestyle Balance Intervention condition [40] used to record physical activity for a one-week period at each assessment and weekly throughout the 12-week intervention period; and Number of Steps were measured during a one-week period at each assessment and throughout the intervention [47].

Physiologic measures included: body mass index (BMI), glycated hemoglobin (HbA1c); fasting blood lipids including HDL-C, total cholesterol, LDL-C (direct), and triglycerides. The maximal graded exercise test (GXT)was utilized to assess maximal aerobic capacity and exercise tolerance at the baseline and POST assessments. In addition, resting, exercise and recovery blood pressures and heart rate, and the Perceived Exertion (Borg Scale) were measured during the GXT to determine perceived stress during aerobic exercise training. Self-monitored blood glucose (SBMG) data was gathered from One-Touch SMBG monitors by participants throughout the study period.

\section{Intervention Design}

The interdisciplinary intervention was composed of 10 manualized CBT sessions, six exercise classes with supervised exercise, and 12 weeks of community-based aerobic activity. These interventions were administered concurrently in order to assess the feasibility of recruiting and 
retaining participants in this demanding combination treatment protocol. CBT sessions were conducted by trained advanced graduate students in the doctoral program in clinical psychology at Ohio University. Sessions were scheduled weekly over the course of the 12-week period, with two weeks available for flexibility in scheduling due to holidays or vacations as needed. The community-based exercise was conducted throughout the 12-week period. An exercise manual, free access to a community exercise facility, and weekly contact with study personnel were provided to participants to encourage adherence to the exercise protocol, collect activity data, and review participant safety and changes to medical status.

Exercise goals were adapted to accommodate the physical and medical restrictions of an olderadult diabetes population. Exercise prescriptions were based on the results obtained from the GXT at baseline. Participants were given exercise goals that represented a total duration of 150 minutes per week with activity that reached 50 to $85 \%$ maximum heart rate consistent with the American College of Sports Medicine recommendations [48, 49]. Due to the limited prior exercise experience of participants as well as the unique needs of patients with depression and diabetes, exercise duration and intensity goals were increased in a graduated fashion during Weeks 1-3, beginning with 100 minutes of weekly exercise and increasing to 150 minutes of total weekly exercise. Participants were asked to complete weekly exercise diaries and to record number of steps measured using pedometers during the intervention period (Weeks 1-12).

\section{Results}

Analysis of demographic characteristics of the total sample $(\mathrm{N}=50)$ enrolled in the intervention indicated that participants had a mean age of 57 (S.D. 9.0) years. The sample was predominantly female (68\%), married (74\%), and evenly distributed across educational levels with a modal 
annual household income of $\$ 21,000-40,000$. The mean duration of T2DM was 11.0 (S.D. 7.0) years. The mean BMI was 35.1 (S.D. 7.1).

Using intent-to-treat analyses, 66\% of participants no longer met criteria for Major Depressive Disorder at POST $(\mathrm{p}<.001)$ and $65 \%$ of participants were remitted at the 3MFU assessment (p<.001). Mean Beck Depression Inventory scores improved significantly both at the POST (8.8, S.D. 8.9, $\mathrm{p}<.05)$ and 3MFU (-8.3, S.D. 10.9, $\mathrm{p}<.05)$ assessments.

With respect to diabetes outcomes, significant decreases in fasting glucose were observed at 3MFU ( $\mathrm{M}=-21.4 \mathrm{mg} / \mathrm{dl}$, S.D. 43.2, $\mathrm{p}<.01)$ as were improvements in LDL-C at POST ( $\mathrm{M}=-11.9$, S.D. 28.6, $\mathrm{p}<.01$ ). Average blood glucose levels (HbA1c) decreased by .32\% at the POST assessment (S.D. 1.0\%, $\mathrm{p}=.05$ ) and $.22 \%$ at the $3 \mathrm{MFU}$ assessment (S.D. $0.9 \%, \mathrm{p}=.10$ ).

Evaluation of exercise outcomes from the GXT procedure showed increased participant capacity for exercise at the POST assessment compared to baseline (VO2 peak M=0.08 L $/ \mathrm{min}$, S.D. 0.2, $\mathrm{p}<.05$ ). Participants completed $193 \mathrm{~min} /$ wk of aerobic activity (range: 76-478) during the intervention, exceeding the graduated study goal of 150 minutes/week. Self-reported physical activity improved significantly from baseline to POST (11.9, S.D. 18.5, $\mathrm{p}<.01$ ) and 3MFU (10.2, S.D. 16.6, $\mathrm{p}<.01$ ). Participants reported significant improvements in their confidence to perform regular physical activity at POST compared to their baseline values (M=5.9, S.D. 13.6, $\mathrm{p}<.05$ ).

Finally, evaluation of diabetes-specific quality of life and social support from baseline to POST and 3MFU assessments showed significant improvements.

\section{Conclusions}


Program ACTIVE demonstrated positive outcomes associated with a rigorous combination behavioral approach to the treatment of depression tailored for individuals living in the rural Appalachian region. Rates of depression remission are comparable to previous studies using single strategy approaches (i.e. CBT, medication, or exercise alone [18, 25]). Improvements in diabetes and exercise outcomes also demonstrated the value of a combination treatment approach for depression, diabetes and cardiovascular risk factors. Moreover, participants reported improvements in quality of life and social support following this demanding intervention.

Barriers endemic to this region such as large geographic distances between place of residence and community facilities, gas price vulnerability, and caregiver demands for family members were identified by participants as pertinent to recruitment and retention in the depression treatment protocol. Participants identified the need for a network of community partners to provide participants with affordable and convenient exercise outlets and cognitive behavioral therapy. Intervention programs such as Program ACTIVE can be successful in improving depression and diabetes outcomes in spite of these barriers if they are flexible in their approach and make use of community resources to facilitate participant self-care. In so doing, there is great opportunity to address the significant costs associated with co-morbid depression and diabetes and to improve the quality of life of residents of this underserved region.

Funding for this study was provided by the National Institutes of Health R34DK71545, Ohio Department of Health, Office of Healthy Ohio, Bureau of Health Promotion and Risk Reduction, Diabetes Prevention and Control Program and the Ohio University Diabetes Research Initiative. This research was conducted with support from the Investigator-Initiated Study Program of Lifescan, Inc. 


\section{References}

Wild, S., Roglic, G., Green, A., Sicree, R., King, H., Global prevalence of diabetes. Estimates for the year 2000 and projections for 2030. Diabetes Care, 2004. 27(5): p. 1047-53.

Schwartz, F., Ruhil, A., Denham, S., Shubrook, J., Simpson, C., Boyd, S. (2009) High selfreported prevalence of diabetes mellitus, heart disease and stroke in eleven counties in rural Appalachian Ohio. J. Rural Health, In press.

American Diabetes Association (2001). Diabetes 2001 Vital Statistics, Alexandria, VA: American Diabetes Association.

Appalachian Regional Commission. (http://www.arc.gov/index.do?nodeId=27; retrieved 1/15/07).

Black, D.A., Sanders, S.G., Labor market performance, poverty, and income inequality in Appalachia. 2004, Appalachian Regional Commission.

Robbins, J.M., Vaccarino, V., Zhang, H., Kasl, S.V., Socioeconomic status and type 2 diabetes in African American and Non-Hispanic White women and men: Evidence from the Third National Health and Nutrition Examination Survey. American Journal of Public Health, 2001. 91(1): p. 76-83.

Weng, C., Coppini, D.V., Sonksen, P.H., Geographic and social factors are related to increased morbidity and mortality rates in diabetic patients. Diabet Med, 2000. 17(8): p. 612-617. 
Robinson, N., Lloyd, C.E., Stevens, L.K., Social deprivation and mortality in adults with diabetes mellitus. Diabet Med, 1998. 15: p. 205-12.

Tang, M., Chen, Y., Krewski, D., Gender-related differences in the association between socioeconomic status and self-reported diabetes. International Journal of Epidemiology, 2003. 32: p. 381-5.

Pincus, T., Callahan, L.F., Burkhauser, R.V., Most chronic diseases are reported more frequently by individuals with fewer than 12 years of formal education in the age 18-64 United States population. J. Chronic Dis, 1987. 40: p. 865-74.

Anderson, R.J., Freedland, K.E., Clouse, R.E., Lustman, P.J., The prevalence of comorbid depression in adults with diabetes: A meta-analysis. Diabetes Care, 2001. 24(6): p. 1069-78.

Lustman, P.J., Anderson, R.J., Freedland, K.E., de Groot, M., Carney, R.M., Clouse, R.E., Depression and poor glycemic control: A meta-analytic review of the literature. Diabetes Care, 2000. 23(7): p. 934-42.

de Groot, M., Anderson, R.J., Freedland, K.E., Clouse, R.E., Lustman, P.J., Association of depression and diabetes complications: A meta-analysis. Psychosomatic Medicine, 2001. 63: p. 619-630.

Clouse, R.E., Lustman, P.J., Freedland, K. E., Griffith, L. S., McGill, J. B., Carney, R. M., Depression and coronary heart disease in women with diabetes. 2002.

Egede, L.E., Diabetes, major depression and functional disability among U.S. adults. Diabetes Care, 2004. 27(2): p. 421-8. 
Ciechanowski, P.S., Katon, W.J., Russo, J.E., Depression and diabetes: Impact of depressive symptoms on adherence, function, and costs. Arch Intern Med, 2000. 160: p. 3278-3285.

Egede, L.E., Zheng, D., Simpson, K., Comorbid depression is associated with increased health care use and expenditures in individuals with diabetes. Diabetes Care, 2002. 25(3): p. 464-470.

Lustman, P.J., Griffith, L.S., Freedland, K.E., Kissel, S.S., Clouse, R.E., Cognitive behavior therapy for depression in type 2 diabetes mellitus. A randomized, controlled trial. Ann Intern Med, 1998. 129(8): p. 613-21.

Lustman, P.J., Griffith, L.S., Clouse, R.E., Freedland, K.E., Eisen, S.A., Rubin, E.H., Carney, R.M., McGill, J.B., Effects of nortriptyline on depression and glycemic control in diabetes: Results of a double-blind, placebo-controlled trial. Psychosom Med, 1997. 59(3): p. 241-50.

Lustman, P.J., Freedland, K.E., Griffith, L.S., Clouse, R.E., Fluoxetine for depression in diabetes: A randomized double-blind placebo-controlled trial. Diabetes Care, 2000. 23(5): p. $618-23$.

Goodnick, P.J., Kumar, A., Henry, J. H., Buki, V. M., Goldberg, R. B., Sertraline in coexisting major depression and diabetes mellitus. Psychopharmacol Bull, 1997. 33(2): p. 261-264.

Lustman, P.J., Griffith, L.S., Clouse, R.E., Freedland, K.E., Eisen, S.A., Rubin, E.H., Carney, R.M., McGill, J.B., Effects of alprazolam on glucose regulation in diabetes. Results of doubleblind, placebo-controlled trial. Diabetes Care, 1995. 18(8): p. 1133-9.

Lustman, P.J., Freedland, K.E., Griffith, L.S., Clouse, R.E., Predicting response to cognitive behavior therapy of depression in type 2 diabetes. Gen Hosp Psychiatry, 1998. 20(5): p. 302-6. 
Lustman, P.J., Clouse, R.E., Treatment of depression in diabetes. Impact of mood and medical outcome. J. Psychosom Res, 2002. 53: p. 917-24.

Blumenthal, J.A., Babyak, M.A., Moore, K.A., Craighead, E., Herman, S., Khatri, P., Waugh, R., Napolitano, M.A., Forman, L.M., Appelbaum, M., Doraiswamy, M., Krishnan, R., Effects of exercise training on older patients with major depression. Arch Intern Med, 1999. 159: p. 23492356.

Babyak, M., Blumenthal, J. A., Herman, S., Khatri, P., Doraiswamy, M., Moore, K., Craighead, E., Baldewicz, T. T., Krishnan, R., Exercise treatment for major depression: Maintenance of therapeutic benefit at 10 months. Psychosom Med, 2000. 62: p. 633-638.

Craft, L.L., Landers, D.M., The effect of exercise on clinical depression and depression resulting from mental illness: A meta-analysis. J. Sport Exer Psychol, 1998. 20: p. 339-57.

Boule, N., Haddad, E., Kenny, G., Wells, G., Sigal, R., Effects of exercise on glycemic control and body mass index in type 2 diabetes: A meta-analysis of controlled clinical trials. JAMA, 2001. 286: p. 1218-1227.

Kirk, A., Mutrie, N., MacIntyre, P., Fisher, M., Effects of a 12-month physical activity counseling intervention on glycemic control and on the status of cardiovascular risk factors in people with type 2 diabetes. Diabetologia, 2004. 47: p. 821-32.

Mourier, A., Gautier, J., Kerviler, E., Mobilization of visceral adipose tissue related to the improvement in insulin sensitivity in response to physical training in NIDDM. Diabetes Care, 1997. 20: p. 385-91. 
Raz, I., Hauser, E., Bursztyn, M., Moderate exercise improves glucose metabolism in uncontrolled elderly patients with non-insulin dependent diabetes mellitus. Isr J. Med. Sci, 1994. 30: p. 766-70.

Rigla, M., Sánchez-Quesada., J.L., Ordóñez-Llanos, J., Prat, Caixàs, A., Jorba, O., Serra, J.R., de Leiva, A., Pérez, A., Effect of physical exercise on lipoprotein(a) and low-density lipoprotein modifications in type 1 and type 2 diabetic patients. Metabolism, 2000. 49(5): p. 640-7.

Lehman R., Engler, H., Honegger, R., Riesen, W., Spinas, G.A., Alterations of lipolytic enzymes and high density lipoprotein subfractions induced by physical activity in type 2 diabetes mellitus. Eur J Clin Invest, 2001. 31: p. 37-44.

Walker, K.Z., Piers, L.S., Putt, R.S., Jones, J.A., O'Dea, K., Effects of regular walking on cardiovascular risk factors and body composition in normoglycemic women and women with Type 2 Diabetes. Diabetes Care, 1999. 22(4): p. 555-61.

Stewart, K., Exercise training and the cardiovascular consequences of type 2 diabetes and hypertension: Plausible mechanisms for improving cardiovascular health. JAMA, 2002. 288(13): p. 1622-31.

American Diabetes Association, Position Statement: Physical Activity/Exercise and Diabetes. Diabetes Care, 2004. 27(1): p. s58-62.

Wierzbicki, A.S., Have we forgotten the pivotal role of high-density lipoprotein cholesterol in atherosclerosis prevention? Current Medical Research and Opinion, 2005. 21(2): p. 299-305. 
Alam, S., Stolinski, M., Pentecost, C., Boroujerdi, M.A., Jones, R.H., Sonksen, P.H., Umpleby, A.M., The effect of a six-month exercise program on very low-density lipoprotein apolipoprotein B secretion in Type 2 diabetes. J. Clin Endo Metab, 2004. 89(2): p. 688-694.

Assman, G., Schulte, H., von Eckardstein, A., Huang, Y., High-density lipoprotein cholesterol as a predictor of coronary heart disease risk. Atherosclerosis, 1996. 124 Suppl.: p. S11-S20.

Diabetes Prevention Program, Diabetes Prevention Program Lifestyle Balance Core Manual. 1996, Pittsburgh, PA: University of Pittsburgh.

Spitzer, R., Williams, J.B.W., Gibbon, M., First, M.B., The structured clinical interview for DSM-III-R (SCID) - I: History, rationale, and description. Arch Gen Psychiatry, 1992. 49: p. 624-629.

Williams, J.B.W., Gibbon, M., First, M. B., Spitzer, R.L., Davies, M., Borus, J., Howes, M.J., Kane, J., Pope, H.G., Jr., Rounsaville, B., Wittchen, H-U., The Structured Clinical Interview for DSM-III-R (SCID) - II. Multisite test-retest reliability. Arch Gen Psychiatry, 1992. 49: p. 630636.

Beck, A.T., Steer, R.A., Brown, G.K., BDI-II Beck Depression Inventory Manual. 2nd Ed. ed. 1996, San Antonio, TX: The Psychological Corporation. Harcourt, Brace \& Company.

Diabetes Control and Complications Trial Research Team, Reliability and validity of a diabetes quality-of-life measure for the Diabetes Control and Complications Trial (DCCT). Diabetes Care, 1988. 11(9): p. 725-732. 
Ware, J., Snow, K., Kosinski, M., Gandek, B., The MOS SF-36 Health Survey: Manual and Interpretation Guide. 1993, Boston, MA: The Health Institute, New England Medical Center.

Glasgow, R.E., Stryker, L.A., Toobert, D.J., Eakin, E., A social-ecological approach to assessing support for disease self-management: The Chronic Illness Resources Survey. J. Behav. Med., 2000. 23(6): p. 559-83.

Melanson, E.L., Knoll, J.R., Bell, M.L., Donahoo, W.T., Hill, J.O., Nysse, L.J., LanninghamFoster, L., Peters, J.C., Levine, J.A., Commercially available pedometers: Considerations for accurate step counting. Preventative Medicine, 2004. 39(2): p. 361-8.

Haskell W.L., Lee, I.M., Pate R.R., Powell K.E., Blair S.N., Franklin B.A., Macera C.A., Heath G.W., Thompson P.D., Bauman A., American College of Sports Medicine; American Heart Association. Physical Activity and Public Health: Updated Recommendation for Adults From the American College of Sports Medicine and the American Heart Association. Circ 116:10811093, 2007.

American College of Sports Medicine, ACSM's Resource Manual for Guidelines for Exercise Testing and Prescription. 4th Ed. ed. 2001, Philadelphia, PA: Lippincott, Williams \& Wilkins. 